\title{
GENERALIZED WAVELET DECOMPOSITIONS OF BIVARIATE FUNCTIONS
}

\author{
CHARLES K. CHUI AND XIN LI
}

(Communicated by Palle E. T. Jorgensen)

\begin{abstract}
The objective of this paper is to introduce an integral transform of wavelet-type on $L^{2}\left(R^{2}\right)$ that can be applied to decompose the space $L^{2}\left(R^{2}\right)$ into a direct sum of subspaces, each of which is identified as $L^{2}(R)$. Projections from $L^{2}\left(R^{2}\right)$ onto these subspaces are also discussed. Moreover, wavelet expansions for functions in $L^{2}\left(R^{2}\right)$ are derived in terms of wavelet bases of $L^{2}(R)$.
\end{abstract}

\section{INTRODUCTION}

Many well-known integral transforms for time-frequency analysis and phasespace consideration take on the formulation

$$
\int_{-\infty}^{\infty} \overline{\lambda(t y)} g(t) d t
$$

where $\lambda$ is a function defined on $(-\infty, \infty)$. These certainly include the Fourier and Laplace transforms. In the analysis of nonstationary signals, for instance, it is sometimes necessary to replace $g(t)$ in (1.1) by functions which depend on more than one variable. In this paper, we study the extension of (1.1) to the formulation

$$
\left(N_{\lambda} f\right)(y):=|y|^{1 / 2} \int_{-\infty}^{\infty} \overline{\lambda(t y)} f(t, y) d t, \quad f \in L^{2}\left(R^{2}\right) .
$$

Here, the kernel $\lambda$ will always be assumed to be in $L^{2}(R)$. Under this assumption, it will be shown that $N_{\lambda}$ is a bounded linear operator from $L^{2}\left(R^{2}\right)$ onto $L^{2}(R)$.

On the other hand, associated with any function $\tilde{\lambda} \in L^{2}(R)$, we introduce another operator $\boldsymbol{M}_{\tilde{\lambda}}$ defined by

$$
\left(M_{\tilde{\lambda}} g\right)(x, y):=|y|^{1 / 2} \tilde{\lambda}(x y) g(y), \quad g \in L^{2}(R) .
$$

Received by the editors August 7, 1992; presented by the second author at the Conference on Interactions Among Wavelets, Control Theory, and Operator Theory, University of North Carolina at Charlotte, May 1, 1993.

1991 Mathematics Subject Classification. Primary 44A15, 46B15; Secondary 41A58, 47B40.

Key words and phrases. Decomposition, integral transforms, linear operators, wavelet transforms.

Research supported by NSF Grants DMS-89-01345, DMS-92-06928 and ARO Contract DAAL03-90-G-0091. 
The importance of these two operators is that they together allow us to construct a "resolution of the identity" that provides a decomposition of the space $L^{2}\left(R^{2}\right)$ as well as a generalization of the integral wavelet transform. More precisely, let $\left\{\lambda_{n}: n \in I\right\}$ be a Riesz basis of $L^{2}(R)$ with dual basis $\left\{\tilde{\lambda}_{n}: n \in I\right\}$ for some countable index set $I$. Here, as usual, duality means that

$$
\left\langle\lambda_{m}, \tilde{\lambda}_{n}\right\rangle:=\int_{-\infty}^{\infty} \lambda_{m}(x) \overline{\tilde{\lambda}_{n}(x)} d x=\delta_{m, n}, \quad m, n \in I .
$$

We will then prove that

$$
f(x, y)=\sum_{n \in I}\left(M_{n} N_{n} f\right)(x, y), \quad f \in L^{2}\left(R^{2}\right),
$$

where, for simplicity, we have used the notation

$$
M_{n}:=M_{\tilde{\lambda}_{n}}, \quad N_{n}:=N_{\lambda_{n}} .
$$

As an example, let $\psi \in L^{2}(R)$ be a wavelet with dual $\tilde{\psi}$ (cf. [1, p. 14]). That is, both

and

$$
\psi_{j, k}(x):=2^{j / 2} \psi\left(2^{j} x-k\right),
$$

$$
\tilde{\psi}_{j, k}(x):=2^{j / 2} \tilde{\psi}\left(2^{j} x-k\right), \quad j, k \in Z,
$$

are Riesz bases of $L^{2}(R)$ such that

$$
\left\langle\psi_{j, k}, \tilde{\psi}_{j^{\prime}, k^{\prime}}\right\rangle=\delta_{j, j^{\prime}} \delta_{k, k^{\prime}}, \quad j, j^{\prime}, k, k^{\prime} \in Z .
$$

Then by considering $I=Z \times Z$ and

$$
\left\{\lambda_{n}\right\}_{n \in I}=\left\{\psi_{j, k}\right\}_{j, k \in I}, \quad\left\{\tilde{\lambda}_{n}\right\}_{n \in I}=\left\{\tilde{\psi}_{j, k}\right\}_{j, k \in I},
$$

the resolution of the identity in (1.4) becomes

$$
\begin{aligned}
f(x, y) & =\sum_{j, k \in Z}|y| \tilde{\psi}_{j, k}(x y) \int_{-\infty}^{\infty} \overline{\psi_{j, k}(s y)} f(s, y) d s \\
& =\sum_{j, k \in Z} \tilde{\psi}_{j, k}(x y) \int_{-\infty}^{\infty} \overline{\psi_{j, k}(t)} f(t / y, y) d t \\
& =\sum_{j, k \in Z} \tilde{\psi}_{j, k}(x y) 2^{j / 2} \int_{-\infty}^{\infty} \overline{\psi\left(2^{j} t-k\right)} f(t / y, y) d t .
\end{aligned}
$$

Here, it is noted that

$$
\left(N_{j, k} f\right)(y):=\left(N_{\psi_{j, k}} f\right)(y)=2^{j / 2}|y|^{-1 / 2} \int_{-\infty}^{\infty} \overline{\psi\left(2^{j} t-k\right)} f(t / y, y) d t
$$

and

$$
\left(M_{j, k} g\right)(x, y):=\left(M_{\tilde{\psi}_{j, k}} g\right)(x, y)=2^{j / 2}|y|^{1 / 2} \tilde{\psi}\left(2^{j} x y-k\right) g(y) .
$$

Of course, the formulation (1.6) extends the notion of the discretized integral wavelet transform (with $y=1$ ) to the upper half-plane (cf. [3, 1, 2]).

To discuss the decomposition of $L^{2}\left(R^{2}\right)$, we consider the range

$$
S_{\tilde{\lambda}}:=\left\{M_{\tilde{\lambda}} g: g \in L^{2}(R)\right\}
$$

of the operator $M_{\tilde{\lambda}}, \tilde{\lambda} \in L^{2}(R)$. It will be shown in the next section that $S_{\tilde{\lambda}}$ is isometrically isomorphic to $L^{2}(R)$. The main result in this paper is 
Theorem 1. Suppose that $\left\{\lambda_{n}: n \in I\right\}$ is a Riesz basis of $L^{2}(R)$ with dual basis $\left\{\tilde{\lambda}_{n}: n \in I\right\}$. Then $L^{2}\left(R^{2}\right)$ has the direct-sum decomposition

$$
L^{2}\left(R^{2}\right)=\bigoplus_{n \in I} S_{n}
$$

where $S_{n}:=S_{\tilde{\lambda}_{n}}$ as defined in (1.8). Moreover, if $\left\{\lambda_{n}: n \in I\right\}$ is an orthonormal basis of $L^{2}(R)$ so that $\tilde{\lambda}_{n}=\lambda_{n}$, then $P_{n}:=M_{n} N_{n}$ is the orthogonal projection from $L^{2}\left(R^{2}\right)$ onto $S_{n}$, and (1.9) becomes an orthogonal decomposition; namely, $S_{n} \perp S_{m}, n \neq m$.

The decomposition of functions of two variables into a sum of functions of one variable was also considered in Jiang and Peng [5], again motivated by the integral wavelet transform and the results in Paul [6].

\section{RESULTS ON INTEGRAL TRANSFORMS OF WAVELET-TYPE}

Before showing that the integral transform $N_{\lambda}$ in (1.2) is a bounded linear operator from $L^{2}\left(R^{2}\right)$ onto $L^{2}(R)$, we make the following simple observation: For any $\tilde{\lambda} \in L^{2}(R)$,

$$
\left\|M_{\tilde{\lambda}} g\right\|_{L^{2}\left(R^{2}\right)}=\|\tilde{\lambda}\|_{L^{2}(R)}\|g\|_{L^{2}(R)}, \quad g \in L^{2}(R) .
$$

Therefore, $M_{\tilde{\lambda}}$ is a linear operator from $L^{2}(R)$ to $L^{2}\left(R^{2}\right)$, and the range $S_{\tilde{\lambda}}$ of $M_{\tilde{\lambda}}$, as defined in (1.8), is a closed subspace of $L^{2}\left(R^{2}\right)$. In particular, if $\|\tilde{\lambda}\|_{L^{2}(R)}=1$, then $S_{\tilde{\lambda}}$ is isometrically isomorphic to $L^{2}(R)$. Furthermore, for any $\lambda, \mu \in L^{2}(R)$, the identity

$$
\left(N_{\lambda} M_{\mu} g\right)(y)=\langle\mu, \lambda\rangle_{L^{2}(R)} g(y), \quad g \in L^{2}(R),
$$

can be easily verified.

Lemma 1. For any $\lambda \in L^{2}(R)$, the operator $N_{\lambda}$ is a bounded linear operator from $L^{2}\left(R^{2}\right)$ onto $L^{2}(R)$ with $\left\|N_{\lambda}\right\|=\|\lambda\|_{L^{2}(R)}$.

Proof. For any $f \in L^{2}\left(R^{2}\right)$,

$$
\begin{aligned}
\left\|N_{\lambda} f\right\|_{L^{2}(R)}^{2} & =\left.\left.\int_{-\infty}^{\infty} d y\left|\int_{-\infty}^{\infty}\right| y\right|^{1 / 2} \overline{\lambda(t y)} f(t, y) d t\right|^{2} \\
& \leq \int_{-\infty}^{\infty} d y\left(|y| \int_{-\infty}^{\infty}|\lambda(t y)|^{2} d t\right) \int_{-\infty}^{\infty}|f(t, y)|^{2} d t \\
& =\|f\|_{L^{2}\left(R^{2}\right)}^{2}\|\lambda\|_{L^{2}(R)}^{2} .
\end{aligned}
$$

By taking $\lambda=\mu$ in (2.2), we have

$$
\left(N_{\lambda} M_{\lambda} g\right)(y)=\|\lambda\|_{L^{2}(R)}^{2} g(y)
$$

for any $g \in L^{2}(R)$. Thus, this lemma is established.

Let $I$ be a countable index set. Suppose that a Riesz basis $\left\{\lambda_{n}: n \in I\right\}$ of $L^{2}(R)$ with dual basis $\left\{\tilde{\lambda}_{n}: n \in I\right\}$ is given. Assume that $\left\{\tilde{\lambda}_{n}: n \in I\right\}$ has Riesz bounds $A$ and $B$; i.e.,

$$
A\left\|\left\{c_{n}\right\}\right\|_{l_{I}^{2}}^{2} \leq\left\|\sum_{n \in I} c_{n} \tilde{\lambda}_{n}\right\|_{L^{2}(R)}^{2} \leq B\left\|\left\{c_{n}\right\}\right\|_{l_{I}^{2}}^{2}
$$


for any $\left\{c_{n}\right\} \in l_{I}^{2}$. It then follows from the expansion

$$
h(x)=\sum_{n \in I}\left\langle h, \lambda_{n}\right\rangle \tilde{\lambda}_{n}(x)=\sum_{n \in I} \tilde{\lambda}_{n}(x) \int_{-\infty}^{\infty} \overline{\lambda_{n}(s)} h(s) d s, \quad h \in L^{2}(R),
$$

that

$$
A \sum_{n \in I}\left|\left\langle h, \lambda_{n}\right\rangle\right|^{2} \leq\|h\|_{L^{2}(R)}^{2} \leq B \sum_{n \in I}\left|\left\langle h, \lambda_{n}\right\rangle\right|^{2} .
$$

Lemma 2. Let $\left\{\lambda_{n}: n \in I\right\}$ be a Riesz basis of $L^{2}(R)$ with dual basis $\left\{\tilde{\lambda}_{n}: n \in\right.$ I\}. Then

$$
f(x, y)=\sum_{n \in I}\left(M_{n} N_{n} f\right)(x, y), \quad f \in L^{2}\left(R^{2}\right),
$$

where the notation in (1.5) is used and the convergence is in $L^{2}\left(R^{2}\right)$.

Proof. Suppose that $h \in L^{2}(R)$ is given. For any $z \neq 0$, let $l(x)=h(x / z)$. Then

$$
\begin{aligned}
h(x) & =l(x z)=\sum_{n \in I} \tilde{\lambda}_{n}(x z) \int_{-\infty}^{\infty} \overline{\lambda_{n}(t)} l(t) d t \\
& =\sum_{n \in I} \tilde{\lambda}_{n}(x z) \int_{-\infty}^{\infty} \overline{\lambda_{n}(t)} h(t / z) d t \\
& =\sum_{n \in I} \tilde{\lambda}_{n}(x z)|z| \int_{-\infty}^{\infty} \overline{\lambda_{n}(s z)} h(s) d s .
\end{aligned}
$$

For any $f \in L^{2}\left(R^{2}\right)$, we have $f(\cdot, y) \in L^{2}(R)$ for almost all $y$, so that

$$
f(x, y)=\sum_{n \in I} \tilde{\lambda}_{n}(x z)|z| \int_{-\infty}^{\infty} \overline{\lambda_{n}(s z)} f(s, y) d s
$$

which becomes $(2.5)$ by taking $z=y$. To complete the proof of the lemma, we need to prove that the convergence is in $L^{2}\left(R^{2}\right)$. So, let us consider an increasing nested sequence of finite subsets $I_{k}$ of $I$ with $\bigcup I_{k}=I$, and consider

$$
g_{k}(y):=\int_{-\infty}^{\infty}\left|f(x, y)-\sum_{n \in I_{k}}\left(M_{n} N_{n} f\right)(x, y)\right|^{2} d x, \quad y \neq 0 .
$$

From (2.6), by taking $z=y$, we have

$$
\lim _{k \rightarrow \infty} g_{k}(y)=0 \text { a.e. }
$$

Write

$$
\begin{aligned}
g_{k}(y) & =\int_{-\infty}^{\infty}\left|f(x, y)-\sum_{n \in I_{k}} \tilde{\lambda}_{n}(x y)\right| y\left|\int_{-\infty}^{\infty} \overline{\lambda_{n}(s y)} f(s, y) d s\right|^{2} d x \\
& =\int_{-\infty}^{\infty}\left|f(x, y)-\sum_{n \in I_{k}} \tilde{\lambda}_{n}(x y) \int_{-\infty}^{\infty} \overline{\lambda_{n}(t)} f(t / y, y) d t\right|^{2} d x
\end{aligned}
$$


Suppose that $A$ and $B$ are Riesz bounds of $\left\{\tilde{\lambda}_{n}: n \in I\right\}$ as described in (2.3). Then, it follows from the Cauchy-Schwarz inequality, (2.3), and (2.4) that

$$
\begin{aligned}
\left(g_{k}(y)\right)^{1 / 2} \leq & \left(\int_{-\infty}^{\infty}|f(x, y)|^{2} d x\right)^{1 / 2} \\
& +\left(\frac{1}{|y|} \int_{-\infty}^{\infty}\left|\sum_{|n| \leq k}\left(f\left(\frac{\dot{y}}{y}, y\right), \lambda_{n}(\cdot)\right) \tilde{\lambda}_{n}(s)\right|^{2} d s\right)^{1 / 2} \\
\leq & \left(\int_{-\infty}^{\infty}|f(x, y)|^{2} d x\right)^{1 / 2}+\left|\frac{B}{y}\right|^{1 / 2}\left(\sum_{|n| \leq k}\left|\left(f\left(\frac{\dot{y}}{y}, y\right), \lambda_{n}(\cdot)\right)\right|^{2}\right)^{1 / 2} \\
\leq & \left(\int_{-\infty}^{\infty}|f(x, y)|^{2} d x\right)^{1 / 2}+\left(\frac{B}{A}\right)^{1 / 2}\left(\frac{1}{|y|} \int_{-\infty}^{\infty}\left|f\left(\frac{x}{y}, y\right)\right|^{2} d x\right)^{1 / 2},
\end{aligned}
$$

or equivalently

$$
g_{k}(y) \leq\left(1+(B / A)^{1 / 2}\right)^{2} \int_{-\infty}^{\infty}|f(x, y)|^{2} d x .
$$

Hence, with (2.8) and (2.9), the Dominated Convergence Theorem applies, yielding

$$
\lim _{k \rightarrow \infty} \int_{-\infty}^{\infty} g_{k}(y) d y=0 .
$$

Since this holds for any $f \in L^{2}\left(R^{2}\right)$, the convergence in (2.5) is in the $L^{2}\left(R^{2}\right)$, completing the proof of the lemma.

We are now ready to prove Theorem 1 .

Proof of Theorem 1. For any $f \in L^{2}\left(R^{2}\right)$, we have, by Lemma $1, N_{n} f \in L^{2}(R)$ so that $M_{n} N_{n} f \in S_{n}$ for any $n \in I$. Therefore, it follows from Lemma 2 that $f \in \sum_{n \in I} S_{n}$, or $L^{2}\left(R^{2}\right)=\sum_{n \in I} S_{n}$. This decomposition of $L^{2}\left(R^{2}\right)$ is actually a direct-sum decomposition. Indeed, if there are two sequences $\left\{g_{n}\right\},\left\{h_{n}\right\} \subset$ $L^{2}(R)$ such that

or equivalently

$$
\sum_{n \in I} M_{n} g_{n}=\sum_{n \in I} M_{n} h_{n}
$$

$$
\sum_{n \in I}|y|^{1 / 2} \tilde{\lambda}_{n}(x y) g_{n}(y)=\sum_{n \in I}|y|^{1 / 2} \tilde{\lambda}_{n}(x y) h_{n}(y),
$$

then we have

$$
\sum_{n \in I} \overline{\lambda_{m}(x y)} \tilde{\lambda}_{n}(x y) g_{n}(y)=\sum_{n \in I} \overline{\lambda_{m}(x y)} \tilde{\lambda}_{n}(x y) h_{n}(y), \quad m \in I .
$$

Integrating both sides of the above identity with respect to $x$ yields $g_{m}=h_{m}$ for all $m \in I$. Hence, the first part of the theorem is proved.

Now if $\left\{\lambda_{n}: n \in I\right\}$ is an orthonormal basis, then it is self-dual. For any $h \in S_{n}$, then $h=M_{n} g$, for some $g \in L^{2}(R)$, and according to (2.2), we have

$$
P_{n} h=M_{n} N_{n}\left(M_{n} g\right)=M_{n}\left(N_{n} M_{n} g\right)=\left\langle\lambda_{n}, \lambda_{n}\right\rangle M_{n} g=h .
$$


On the other hand, for any $f$ orthogonal to $S_{n}$ and $\beta \in L^{2}\left(R^{2}\right)$, we have

$$
\begin{aligned}
\left\langle M_{n}\right. & \left.N_{n} f, \beta\right\rangle_{L^{2}\left(R^{2}\right)} \\
& =\int_{-\infty}^{\infty} d y \int_{-\infty}^{\infty}\left(|y| \lambda_{n}(x y) \int_{-\infty}^{\infty} \overline{\lambda_{n}(s y)} f(s, y) d s\right) \overline{\beta(x, y)} d x \\
& =\int_{-\infty}^{\infty} d y \int_{-\infty}^{\infty} f(s, y) \overline{\left(|y| \lambda_{n}(s y) \int_{-\infty}^{\infty} \overline{\lambda_{n}(x y)} \beta(x, y) d x\right)} d s \\
& =\left\langle f, M_{n} N_{n} \beta\right\rangle_{L^{2}\left(R^{2}\right)}=0 .
\end{aligned}
$$

Hence, $P_{n}=M_{n} N_{n}$ is the orthogonal projection from $L^{2}\left(R^{2}\right)$ onto $S_{n}$. Finally, for any $h_{1} \in S_{m}, h_{2} \in S_{n}, m \neq n$, we have $h_{1}=S_{m} g_{1}, h_{2}=S_{n} g_{2}$, for some $g_{1}, g_{2} \in L^{2}(R)$, and

$$
\begin{aligned}
\left\langle h_{1}, h_{2}\right\rangle_{L^{2}\left(R^{2}\right)} & =\int_{-\infty}^{\infty} d y \int_{-\infty}^{\infty}\left(M_{n} g_{1}\right)(x, y) \overline{\left(M_{m} g_{2}\right)(x, y)} d x \\
& =\int_{-\infty}^{\infty} d y \int_{-\infty}^{\infty}|y| \lambda_{n}(x y) \overline{\lambda_{m}(x y)} g_{1}(y) \overline{g_{2}(y)} d y \\
& =\int_{-\infty}^{\infty} g_{1}(y) \overline{g_{2}(y)} d y \int_{-\infty}^{\infty} \lambda_{n}(x) \overline{\lambda_{m}(x)} d x=0 .
\end{aligned}
$$

This completes the proof of the theorem.

More generally, even if $\left\{\lambda_{n}: n \in I\right\}$ is not orthonormal but only a Riesz basis of $L^{2}(R)$ with dual $\left\{\tilde{\lambda}_{n}: n \in I\right\}$, it is easy to verify that the operator $P_{n}=M_{n} N_{n}$ is an affine projection from $L^{2}\left(R^{2}\right)$ onto $S_{n}$ in the sense that $P_{n} f=f$ for any $f \in S_{n}$, and $P_{n} f=0$ for all $f \in S_{m}$, where $m \neq n$.

We end this paper by making two remarks.

(1) The decomposition result in Theorem 1 can be applied in signal analysis to decompose a nonstationary signal $f(w, t)$, where $w$ is the frequency variable. By selecting an appropriate Riesz basis $\left\{\lambda_{n}: n \in I\right\}$ with dual $\left\{\tilde{\lambda}_{n}: n \in I\right\}$, the signal $f(w, t)$ is mapped to a sequence of stationary signals

$$
g_{n}(t):=\left(N_{n} f\right)(t) \in L^{2}(R), \quad n \in I,
$$

with certain desirable properties. Of course, the original signal can be reconstructed from $\left\{g_{n}: n \in I\right\}$ by using the identity

$$
f(w, t)=\sum_{n \in I}\left(M_{n} g_{n}\right)(w, t) .
$$

The schemetic diagram for this decomposition and reconstruction procedure is shown in the following figure, where $I=\left\{n_{i}: i \in Z\right\}$ :

$$
f(w, t) \longrightarrow g_{n_{n_{i-1}}(t) \rightarrow M_{n_{n_{i-1}}}} \rightarrow g_{n_{i}}(t) \rightarrow M_{n_{i}}
$$

In practice, the components $g_{n_{i}}$ of $f$ are "filtered" by certain operators $H_{n_{i}}$. For instance, Hankel-type operators $H_{n_{i}}$ can be used for problems in 
systems theory such as systems reduction and identification. Of course, the reconstruction operators $M_{n_{i}}$ are then applied to $H_{n_{i}} g_{n_{i}}$ instead of $g_{n_{i}}$.

(2) It is also worth mentioning that nonstationary signals are obtained by applying the Zak transform to stationary signals, namely,

$$
f(w, t)=(Z g)(t, w):=a^{1 / 2} \sum_{k \in Z} g(t a+k a) e^{2 \pi i k w}, \quad g \in L^{2}(R) .
$$

This transformation was introduced by Zak [7] in the solid state physics literature. It can easily be verified that the Zak transform is a unitary map from $L^{2}(R)$ into $L^{2}([0,1) \times[0,1))$. For more details, the interested reader is referred to $[7,4]$.

\section{REFERENCES}

1. C. K. Chui, An introduction to wavelets, Academic Press, New York, 1992.

2. I. Daubechies, Ten lectures on wavelets, CBMS-NSF Regional Conf. Ser. in Appl. Math., vol. 61, SIAM, Philadelphia, PA, 1992.

3. A. Grossmann and J. Morlet, Decomposition of Hardy functions into square integrable wavelets of constant shape, SIAM J. Math. Anal. 15 (1984), 723-736.

4. C. Heil and D. F. Walnut, Continuous and discrete wavelet transforms, SIAM Rev. 31 (1989), 628-666.

5. Q. Jiang and L. Peng, Wavelet transform and Ha-plitz operators, Research Report no. 5, Inst. Math. and Dept. of Math., Peking Univ., 1991.

6. T. Paul, Functions analytic on the half-plane as quantum mechanical states, J. Math. Phys. 25 (1985), 3252-3263.

7. J. Zak, Finite translations in solid state physics, Phys. Rev. Lett. 19 (1967), 1385-1397.

Department of Mathematics, Texas A\&M University, College Station, Texas 778433368

E-mail address: cchui@tamu.edu

Department of Mathematical Sciences, University of Nevada, Las Vegas, Nevada 89154

E-mail address: xinlixin@nevada.edu 